\title{
Development of Cancer Vaccines Targeting Brachyury, a Transcription Factor Associated with Tumor Epithelial-Mesenchymal Transition
}

\author{
Duane H. Hamilton Justin M. David Charli Dominguez Claudia Palena \\ Laboratory of Tumor Immunology and Biology, Center for Cancer Research, National Cancer Institute, \\ National Institutes of Health, Bethesda, MD, USA
}

\section{Keywords}

Epithelial-mesenchymal transition · Brachyury - Cancer vaccines $\cdot$ Immune resistance

\begin{abstract}
Epithelial-mesenchymal transition (EMT) is recognized as a relevant process during the progression of carcinomas towards metastatic disease. Epithelial cancer cells undergoing an EMT program may acquire mesenchymal features, motility, invasiveness, and resistance to a variety of anticancer therapeutics. Preventing or reverting the EMT process in carcinomas has the potential to minimize tumor dissemination and the emergence of therapeutic resistance. One of the strategies currently under investigation to target tumor cells undergoing EMT is the generation of a sustained immune response directed against an essential molecular driver of the process. This review focuses on the current development of immune-mediated anticancer interventions aimed at targeting a transcription factor, brachyury, associated with human tumor EMT. Also presented here is a summary of recent studies demonstrating a role for EMT in tumor resistance to immune effector cytotoxicity, and the study of novel strategies aimed at reverting the EMT to be used in combination with immune-mediated anticancer interventions.
\end{abstract}

(c) 2017 S. Karger AG, Basel

\section{Introduction}

Epithelial-mesenchymal transition (EMT) is recognized as a relevant process during the progression of carcinomas towards metastatic disease [Thiery, 2002; Kalluri and Weinberg, 2009]. While EMT is normally involved in the morphogenetic events of embryogenesis, evidence from preclinical models and clinical samples [Creighton et al., 2009; Yu et al., 2013; Tan et al., 2014] has led to the understanding that epithelial cancer cells may also activate an EMT program, resulting in the acquisition of mesenchymal features, motility, invasiveness, and resistance to a variety of anticancer therapeutics. Preventing or reverting the EMT process in carcinomas thus represents an attractive modality to minimize tumor dissemination and the emergence of therapeutic resistance.

\begin{tabular}{ll}
\hline Abbreviations used in this paper \\
\hline Ad5 & adenovirus serotype 5 \\
EMT & epithelial-mesenchymal transition \\
HLA & human leukocyte antigen \\
MVA & modified vaccinia strain Ankara \\
NK & natural killer cells
\end{tabular}


In light of recent advances in the field of cancer immunotherapy, one therapeutic strategy to address the problem of EMT is the generation of a sustained immune response directed against essential molecular drivers of the process. This review is focused on the current status of development of immune-mediated anticancer interventions aimed at targeting a transcription factor, brachyury, associated with tumor EMT. Among the topics discussed here are: (a) the characterization of brachyury as a tumor-associated molecule and its potential function in the biology of the tumor, (b) the potential role of EMT in mechanisms of resistance to immune attack, and (c) the potential for the mitigation of metastatic disease by the use of combinatorial therapies of cancer vaccines and other agents that alleviate tumor EMT. While this review does not discuss in detail the current knowledge about the occurrence and the role of EMT in cancer, comprehensive reviews on this topic can be found in the literature [Thiery, 2002, 2003; Kalluri and Weinberg, 2009].

\section{Tumor-Associated Pattern of Brachyury Expression}

The T-box transcription factor brachyury (encoded by the $T$ gene) plays an essential role in the formation of the posterior mesoderm in the vertebrate embryo [Herrmann et al., 1990; Kispert and Herrmann, 1993; Kispert et al., 1995; Edwards et al., 1996]. Studies performed mainly in mouse or rat model systems have shown that, early in development, brachyury is transiently expressed in mesodermal precursor cells within (and adjacent to) the primitive streak [Wilkinson et al., 1990], subsequently downregulated in late-stage embryos, and is eventually undetectable in the majority of adult normal tissues. The only sites where brachyury expression has been so far reported in late-development and adult normal tissues are the immature notochordal cells of the intervertebral discs in mice and rats [Wilkinson et al., 1990; Tang et al., 2012], scattered cells in the adult human seminiferous tubules [Miettinen et al., 2015], and isolated cells in normal adult human thyroid [Roselli et al., 2012]. This highly restrictive pattern of expression along normal development contrasts with that of neoplastic growth, as high levels of brachyury can be detected in a range of human tumor types (Table 1). The initial reports of brachyury overexpression in cancer originated from studies in chordoma, a rare malignant tumor that occurs along the spine and is believed to originate from remnants of the notochord. High levels of brachy-
Table 1. Expression of brachyury in various types of human tumors

\begin{tabular}{ll}
\hline Tumor type & References \\
\hline Chordoma & $\begin{array}{l}\text { Vujovic et al., 2006; Tirabosco et al., } \\
2008\end{array}$ \\
Germ cell & Miettinen et al., 2015 \\
Hemangioblastoma & Barresi et al., 2012 \\
GIST - gastrointestinal & Pinto et al., 2015 \\
stromal tumors & Roselli et al., 2012; Haro et al., 2013 \\
Lung carcinoma & $\begin{array}{l}\text { Miettinen et al., 2015 } \\
\text { Small cell carcinoma, lung } \\
\text { Breast carcinoma }\end{array}$ \\
Colon carcinoma & Kilic et al., 2011 \\
Hepatocellular carcinoma & Du et al., 2014 \\
Prostate carcinoma & Pinto et al., 2014 \\
Oral squamous carcinoma & Imajyo et al., 2012 \\
\hline
\end{tabular}

ury protein can be observed in almost $100 \%$ of chordomas [Vujovic et al., 2006; Tirabosco et al, 2008], and a recent report identified $T$ (brachyury) gene duplication as a major susceptibility factor for familial chordoma [Yang et al., 2009]. Expression of brachyury in tumors of epithelial origin was initially identified by computerbased differential display analysis of expressed sequence tag clusters in the UniGene human database [Palena et al., 2007]. Brachyury mRNA was found to be highly represented among tumor-derived versus normal tissue-derived libraries, an observation later confirmed and expanded at the protein level with a variety of human tumors. Brachyury protein has now been shown to be aberrantly overexpressed in tumors of the lung, including in $50 \%$ of adenocarcinomas, $25 \%$ of squamous carcinomas [Roselli et al., 2012; Haro et al., 2013; Hamilton et al., 2015], and $40 \%$ of small cell carcinomas [Miettinen et al., 2015], breast [Palena et al., 2014; Shao et al., 2015], colon [Kilic et al., 2011], prostate [Pinto et al., 2014], and hepatocellular carcinomas [Du et al., 2014]. In the case of lung, breast, and prostate cancer, expression of brachyury has been observed not only in primary tumors, but also in metastatic lesions, including both tumor-positive lymph nodes and distant metastases. Interestingly, various immunohistochemical studies have shown that the localization of the brachyury protein varies among tumor types. Brachyury expression is nuclear in chordomas and cytosolic in hemangioblastomas [Tirabosco et al., 2008; Barresi et al., 2012; Barresi et al., 2014], while carcinomas show brachyury protein localized either in the nucleus and/or the cytosol of the tumor cells [Roselli et al., 2012; Pinto et al., 2014]. 


\section{Brachyury and EMT}

Using multiple experimental systems, it has been demonstrated that overexpression of the transcription factor brachyury in carcinoma cells promotes their transition from an epithelial to a mesenchymal-like phenotype in vitro [Fernando et al., 2010; Fernando et al., 2011; Larocca et al., 2013]. This was first described by Fernando et al. [2010] with human lung and pancreatic carcinoma cells, where overexpression of brachyury was associated with the upregulation of mesenchymal proteins, loss of epithelial markers (including E-cadherin), and gain of motility and invasiveness in vitro. Brachyury overexpression was shown to decrease the transcriptional activity of the Ecadherin promoter in cancer cells, via its binding to a half T-box DNA binding site in the promoter of E-cadherin, and cooperation with the repressor Snail2. In vivo, silencing of brachyury in lung carcinoma cells did not affect primary tumor growth, but brachyury-silenced cells demonstrated a reduced ability to disseminate from the primary subcutaneous tumor to the site of metastasis. Furthermore, these cells also exhibited a reduced ability to form experimental lung metastasis after intravenous implantation [Fernando et al., 2010]. An association of brachyury with the occurrence of EMT in human tumor cells has now been shown with hepatocellular [Du et al., 2014], breast [Palena et al., 2014; Shao et al., 2015] and prostate [Pinto et al., 2014] carcinoma cells in culture. In all of these studies, overexpression of brachyury induced the expression of mesenchymal markers, migration, and invasion, while decreasing expression of epithelial proteins in carcinoma cells.

\section{Brachyury and EMT Markers: Association in vivo}

In general, the occurrence of EMT and its molecular drivers in human carcinomas in vivo has been scarcely reported compared to the extensive amount of data describing the manifestation of EMT in preclinical models in vitro. Brachyury is not the exception, as very few studies so far have investigated the expression of this molecule in human tumor biopsies in the context of markers of EMT. In a recent report [Xu et al., 2015], expression of brachyury, E-cadherin, and $\mathrm{N}$-cadherin were compared by immunohistochemistry in $>100$ non-small cell lung cancer tissue samples. Brachyury upregulation was demonstrated in $40 \%$ of lung tumors and an inverse association was observed between brachyury and E-cadherin, whereas $\mathrm{N}$ cadherin expression was increased in brachyury-positive cases. In another study with prostate cancer tissues, expression of brachyury mRNA was correlated with the expression of genes associated with EMT [Pinto et al., 2014], once again demonstrating a significant positive association between brachyury and the mesenchymal-associated markers fibronectin, Snail1, TGF- $\beta 1$, IL-8, MMP14, and MMP24, and an inverse correlation between brachyury and epithelial E-cadherin. Although limited, the evidence from these clinical studies suggests a potential involvement of brachyury in tumor EMT in vivo.

\section{Brachyury and Tumor Resistance}

As a consequence of transitioning from an epithelial to a mesenchymal phenotype, tumor cells adopt the ability to overcome a variety of cell death-inducing signals [Vega et al., 2004; Kurrey et al., 2009], thus becoming resistant to a range of cytotoxic therapies, including chemotherapy, radiation, epidermal growth factor receptor kinase inhibitors [Thomson et al., 2005; Thomson et al., 2008; Byers et al., 2013], and HER-2-directed therapy [Kim et al., 2014]. With brachyury in particular, it has been shown that increased levels of expression in lung cancer cell lines associate with increased resistance to cisplatin, vinorelbine, docetaxel, and radiation in vitro. Interestingly, reducing brachyury expression in tumor cells either directly via a brachyury-targeting shRNA or indirectly through inhibition of the TGF- $\beta$ signaling pathway resulted in increased tumor responsiveness to chemotherapy. In addition, chemotherapy treatment of lung cancer cells in vitro and in vivo has been shown to select for a population of tumor cells with high levels of brachyury [Huang et al., 2013; Larocca et al., 2013]. These observations were subsequently expanded to human breast cancer cells, where overexpression of brachyury resulted in lower cellular proliferation rates and acquisition of resistance to docetaxel [Palena et al., 2014].

\section{Brachyury and Tumor Prognosis}

High levels of brachyury mRNA or protein in the primary tumor have been shown to associate with poor clinical outcome in patients with various tumor types. In lung cancer, for example, the level of brachyury mRNA expression in the primary tumor was shown to significantly predict poor prognosis in both 5-year disease-free survival and overall survival analyses, as well as to positively correlate with vascular and lymphatic invasion and tu- 
mor grade and stage [Haro et al., 2013]. Similarly, expression of brachyury protein has been positively correlated with decreased survival in early-stage colorectal cancer patients [Kilic et al., 2011], with a high rate of metastasis and poor prognosis of hepatocellular carcinoma [Du et al., 2014], and with poor survival in patients with oral squamous cell carcinoma [Imajyo et al., 2012]. Our group has also analyzed the risk of recurrence associated with low, medium, and high levels of brachyury mRNA in the primary tumor of $>300$ breast cancer patients treated for 5 years with tamoxifen only as adjuvant therapy. The results of this analysis demonstrated that high brachyury levels are significantly associated with a higher risk of recurrence and distant metastasis [Palena et al., 2014]. Interestingly, the association between brachyury and prognosis in breast cancer was not observed among patients who were treated with surgery/local therapy alone, thus suggesting that brachyury might be associated with tamoxifen resistance in breast cancer, rather than related to progression mechanisms independent of hormone-receptor treatment. In prostate cancer, brachyury protein expression has also been associated with tumor progression and aggressiveness, as brachyury-positive cases corresponded with highly undifferentiated tumors and a higher Gleason score [Pinto et al., 2014].

\section{Immunotherapeutic Approaches against EMT}

The cancer immunotherapy field has recently experienced a revival due to the unprecedented rates of durable tumor responses observed in melanoma [Topalian et al., 2014], lung [Borghaei et al., 2015; Garon et al., 2015], and bladder cancer patients [Powles et al., 2014], among others, following treatment with monoclonal antibodies targeting the PD-1 (programmed cell death-1) protein or the CTLA-4 (cytotoxic T-lymphocyte-associated protein 4) immune checkpoint receptors. Such treatments, which act by disinhibiting tumor-reactive immune responses, can result in a marked reduction of tumor burden in some patients [Shin and Ribas, 2015].

One approach by which to target tumor cells undergoing an EMT is to immunize a patient against 1 (or more) EMT driver(s) to elicit or enhance an effective T-cell immune response that will selectively recognize and eradicate tumor cells expressing the EMT driver of choice. To be considered as a viable target, the EMT driver must fulfill at least 2 requisites: (1) have a tumor-restricted pattern of expression in adult tissues, and (2) be immunogenic (i.e. able to induce an immune response).

Brachyury-Based Cancer Vaccines

\section{Brachyury as a Tumor Antigen}

Immunization against a tumor antigen is expected to elicit a long-term memory $\mathrm{T}$-cell response against the antigen. To avoid potential autoimmunity, the tissue distribution of the target antigen(s) is an important consideration. As described above, the transcription factor brachyury has a pattern of expression that is restricted primarily to neoplastic tissues in the adult, thus satisfying the first requisite as a target for immune intervention. In terms of immunogenicity, the ability of brachyury to activate an immune response has been shown first with preclinical models both in vitro and in vivo and, more recently, in patients vaccinated with brachyury-based vaccines, as discussed below. In the initial preclinical studies [Palena et al., 2007], a human leukocyte antigen (HLA)- $\mathrm{A}_{0201}$-restricted epitope of brachyury (WLLPGTSTL) identified via the use of an HLA-binding prediction algorithm was used in vitro to stimulate $\mathrm{T}$ cells derived from the blood of cancer patients. The expanded brachyury-specific CD8+ T cells were able to lyse carcinoma cells of lung, colorectal, and breast origin that expressed the brachyury protein in an HLA-A $\mathrm{A}_{0201}$ restricted fashion [Palena et al., 2007; Roselli et al., 2012; Palena et al., 2014]. This peptide was later modified to improve its binding to the HLA$\mathrm{A}_{0201}$ molecule by substitution of the amino acid at the anchor residue at position 9 from leucine to valine (WLLPGTSTV), resulting in an enhanced ability to stimulate brachyury-specific $\mathrm{T}$ cells in vitro and in vivo in HLA-A2 transgenic mice [Tucker et al., 2014].

The inherent immunogenicity of brachyury was also revealed from the analysis of immune responses in cancer patients immunized against carcinoembryonic antigen or prostate-specific antigen. In addition to generating responses against the tumor-associated antigens contained within their respective vaccines, development of brachyury-reactive CD8+ T cells was also observed [Madan et al., 2012]. This expansion of brachyury-specific T cells may have been the result of cross-presentation of the antigen to the immune system, following tumor destruction in response to the vaccine. These studies demonstrated that brachyury is immunogenic, and has the potential to function as a target for anticancer vaccination.

\section{Therapeutic Vaccines against Brachyury}

There are several different vaccine-delivery modalities aimed at eliciting a tumor-specific T cell-mediated immune response. These include recombinant vectors, peptide- and 
protein-based vaccines, dendritic cell-based vaccines, and whole tumor cell vaccines [Palena and Schlom, 2010]. Currently, 3 cancer vaccine platforms have been developed to target the transcription factor brachyury: recombinant yeast-, poxvirus-, and adenovirus-based vaccines.

\section{Yeast-Based Brachyury Vaccine}

Yeast vectors consist of heat-killed recombinant Saccharomyces cerevisiae engineered to express 1 or more tumor antigens. Yeast vaccines can be easily propagated, stored, and administered as "off-the-shelf" agents, and have been used in multiple clinical trials in patients with various carcinomas [Bilusic et al., 2014; Chaft et al., 2014], demonstrating excellent safety and the ability to induce measurable immune responses against the tumor antigen encoded by the yeast vector. A heat-killed recombinant yeast expressing the full-length human brachyury protein has been developed (designated as GI-6301). In preclinical in vitro studies, this vaccine has been used to activate and promote the maturation of human dendritic cells as well as to activate and expand brachyury-specific CD8+ and CD4+ T cells from the blood of healthy donors and cancer patients [Hamilton et al., 2013]. Experiments conducted in vivo also demonstrated that vaccination of C57BL/6 mice with yeast-brachyury (and not a control vaccine) could elicit brachyury-specific CD8+ and CD4+ T-cell responses that significantly reduced the tumor burden in an experimental model of lung metastasis [Hamilton et al., 2013; Palena et al., 2014] in the absence of toxicity.

Based on these preclinical investigations, a phase I clinical trial of the yeast-brachyury vaccine was initiated and recently completed in patients with advanced cancer [Heery et al., 2015]. The study was aimed at evaluating the safety of escalating doses of the brachyury-based vaccine and included 34 patients, 23 with advanced carcinomas and 11 with chordoma. The yeast-brachyury vaccine was well tolerated with no associated toxicities. Analysis of immune responses in the blood of 31 vaccinated patients utilizing a pool of 15-mer peptides reflecting the entire sequence of brachyury demonstrated the presence of brachyury-specific CD8+ and/or CD4+ T-cell responses in approximately $50 \%$ of patients postvaccination, including all dose levels. In some of these patients, brachyury-specific T cells could be detected in the blood prevaccination and were enhanced postadministration of the yeast-brachyury vaccine. These results demonstrated, for the first time in humans, that a transcription factor involved in the process of tumor EMT could be immuno- logically targeted via vaccination. Some evidence of clinical activity was also reported in this trial, with 1 chordoma patient having a mixed response and another chordoma patient a confirmed partial response. Based on these results, a randomized phase II clinical trial of the yeast-brachyury vaccine is currently ongoing in patients with chordoma randomized to receive radiation with or without the vaccine (https://www.clinicaltrials.gov/ct2/ show/NCT02383498).

\section{Poxvirus-Based Brachyury-TRICOM Vaccine}

Poxviruses, including the avipox virus fowlpox, vaccinia, and modified vaccinia strain Ankara (MVA) are being explored in numerous clinical trials as vaccine-delivery systems [Madan et al., 2012]. MVA is a highly attenuated strain of vaccinia virus that is nonreplicative in human cells, and has been safely administered to a large number of individuals as part of the smallpox eradication campaign, including individuals with immune deficiencies [Mayr and Danner, 1978]. One of the advantages of poxviral vectors is that they can accept large inserts of DNA and thus transgenes for 1 or more tumor antigens and costimulatory molecules can be introduced. After poxviral infection of antigen-presenting cells at the site of injection, the encoded transgenes can be processed and presented by both MHC-class I and II pathways in the infected cells, leading to the activation of CD8+ and CD4+ T-cell responses.

Optimal activation of $\mathrm{T}$ cells is known to require 2 signals. The first is mediated by the interaction of the T-cell receptor on the T cells with the peptide/MHC complex on the surface of the target cell. The second signal, called costimulation, is provided by the interaction of accessory molecules on the surface of the antigen-presenting cells and their ligands on the T cells. The combination of several costimulatory molecules on a vaccine delivery vector has been demonstrated to further enhance the activation of T-cell responses specific against the tumor antigen(s) encoded by the vaccine. One such combination, called TRICOM (including the costimulatory molecules B7-1, ICAM-1, and LFA-3), has been extensively tested in the clinic as part of several vaccine platforms [Madan et al., 2012]. An MVA-poxviral vaccine vector encoding the full-length human brachyury and the triad of costimulatory molecules, TRICOM, has been developed and is currently being tested in a phase I clinical trial in patients with advanced carcinomas (http://www.clinicaltrials. gov/ct2/show/NCT02179515). 


\section{Adenovirus-Based Brachyury Vaccine}

A different cancer vaccine delivery system consists of an adenovirus serotype 5 (Ad5), designated as Ad5[E1-, E2b-], in which regions of the early 1 (E1), early 2 (E2b), and early 3 (E3) Ad5 genes have been deleted, leading to a marked reduction in the host immune response to the vector [Gabitzsch et al., 2009]. This platform has been used in the clinic to demonstrate the ability to induce target-specific immune responses in the absence of toxicities [Gabitzsch et al., 2010]. Recently, an Ad5-brachyury was constructed to encode the entire brachyury gene devoid of 25 amino acids involved in DNA binding and modified to express an enhanced T-cell epitope [Gabitzsch et al., 2015]. This vector is part of a triad of admixed adenoviruses, designated Tri-Ad5, consisting of Ad5 vectors encoding for 1 of 3 tumor antigens: brachyury, CEA (carcinoembryonic antigen) and MUC1 (mucin-1). Tri-Ad5 was used in preclinical studies with human $\mathrm{T}$ cells and in vivo in C57BL/6 mice, demonstrating the ability to expand $\mathrm{T}$ cells specific for the 3 encoded tumor antigens, including the transcription factor brachyury.

Because multiple tumor types show some level of brachyury expression, we anticipate that the brachyurybased vaccines so far developed could potentially be used as therapeutics for a range of cancer types.

\section{Mechanisms of Tumor Escape to Immune Attack}

Tumor cells are able to grow and metastasize in spite of the presence of measurable antitumor-specific immune responses, an observation that led to the inclusion of "immune-evasion" as an "emerging hallmark of cancer" [Hanahan and Weinberg, 2011]. Multiple mechanisms may contribute to the ability of tumor cells to evade a tumor-specific immune response, including defects in the antigen presentation, the presence of immune suppressive mediators, and/or regulatory cells in the microenvironment of the tumor, and the ability to overcome apoptosis triggered by immune effector cytotoxic mechanisms. Comprehensive reviews can be found in the literature extensively describing the multiple mechanisms of immune resistance employed by cancer cells [Zitvogel et al., 2006; Whiteside, 2009; Ribas, 2015].

While the effect of tumor EMT in resistance to conventional anticancer interventions or to certain targeted therapies has been extensively studied [Vega et al., 2004; Thomson et al., 2008; Huang et al., 2013], the number of studies regarding the potential role of EMT in tumor es- cape from immune surveillance and rejection is limited. One of the first reports on the negative impact of EMT in antitumor immunity has demonstrated that melanoma cells transfected to overexpress the EMT driver Snail are able to induce an immune suppressive environment that promotes metastasis via the accumulation of Treg (T regulatory) Foxp3+CD4+ T cells [Kudo-Saito et al., 2009]. The authors showed that Snail-expressing cancer cells secrete high levels of TGF- $\beta 1$ and thrombospondin- 1 that, in turn, could induce CD4+ T cells into Tregs. In vivo, Snail+ melanoma cells were resistant to immunotherapy with an intratumoral injection of dendritic cells pulsed with a tumor antigen, and overexpression of Snail was found to drive the resistance of tumor cells to cytotoxicity mediated by antigen-specific T lymphocytes, as compared to Snail-negative tumor cells, in vitro.

Subsequent studies by Akalay et al. [2013a, b] analyzed the mechanism(s) involved in the ability of tumor cells undergoing EMT to overcome T cell-mediated lysis. In their studies, various derivatives of breast MCF-7 cells transfected with Snaill or undergoing EMT via the acquisition of resistance to TNF- $a$ were compared for their susceptibility to lysis by a cytotoxic T-cell clone. The results demonstrated that MCF7 cells that underwent an EMT were significantly less susceptible to T cell-mediated lysis via the activation of authophagy pathways, as shown by the increased formation of autophagosomes and increased coexpression of markers of autophagy in EMT versus control tumor cells.

Another example of the negative impact of tumor EMT in immune-mediated lysis originated from the study of carcinoma cells with very high levels of the transcription factor brachyury. Our group has analyzed the susceptibility to cytotoxicity of various human carcinoma cell lines with a range of brachyury expressions (Fig. 1a) in response to brachyury-specific CD8+ cytotoxic Tlymphocytes. The results of this analysis unexpectedly showed a bell-shape response curve, where tumor cells with very low or very high levels of the target brachyury were only minimally susceptible to lysis by the brachyury-specific $\mathrm{T}$ cells. As antigen-specific T cells require some level of the antigen (in this case, brachyury) expressed on the target cells to mediate their lysis, the low lysis observed with cancer cells expressing very low levels of the antigen brachyury was expected. However, it was unexpected to observe that in the presence of very high levels of the target, the brachyury-specific $T$ cells mediated a lower degree of lysis. This observation was expanded utilizing lung epithelial H441 cells, which endogenously express low levels of brachyury and are lysed by brachyury-spe- 
Fig. 1. Very high levels of brachyury expression may impair the immune-mediated cytotoxicity of carcinoma cells. a Expression of brachyury mRNA relative to that of GAPDH in various human carcinoma cell lines (green line) was compared to their susceptibility to lysis mediated by brachyury-specific CD8+ T cells (blue line). All carcinoma cell lines used in this assay were HLA-A $\mathrm{A}_{02}$ positive and were lysed by brachyury-specific CD8+ T cells recognizing an HLA- $\mathrm{A}_{0201}$-restricted epitope of brachyury. PANC-1 pBr corresponds to PANC-1 cells transfected to overexpress brachyury. b Lysis of lung carcinoma $\mathrm{H} 441$ cells by brachyury-specific or MUC1-specific CD8+ T cells. H441 cells were transfected with a control pCMV vector, a vector encoding full-length human brachyury (pBr), a control nontargeting siRNA (Con siRNA), or a brachyury-silencing pool of siRNA (Br siRNA). ${ }^{* *} p<$ 0.05 for the comparison of $\mathrm{pBr}$ versus pCMV, and Br siRNA versus Con siRNA.

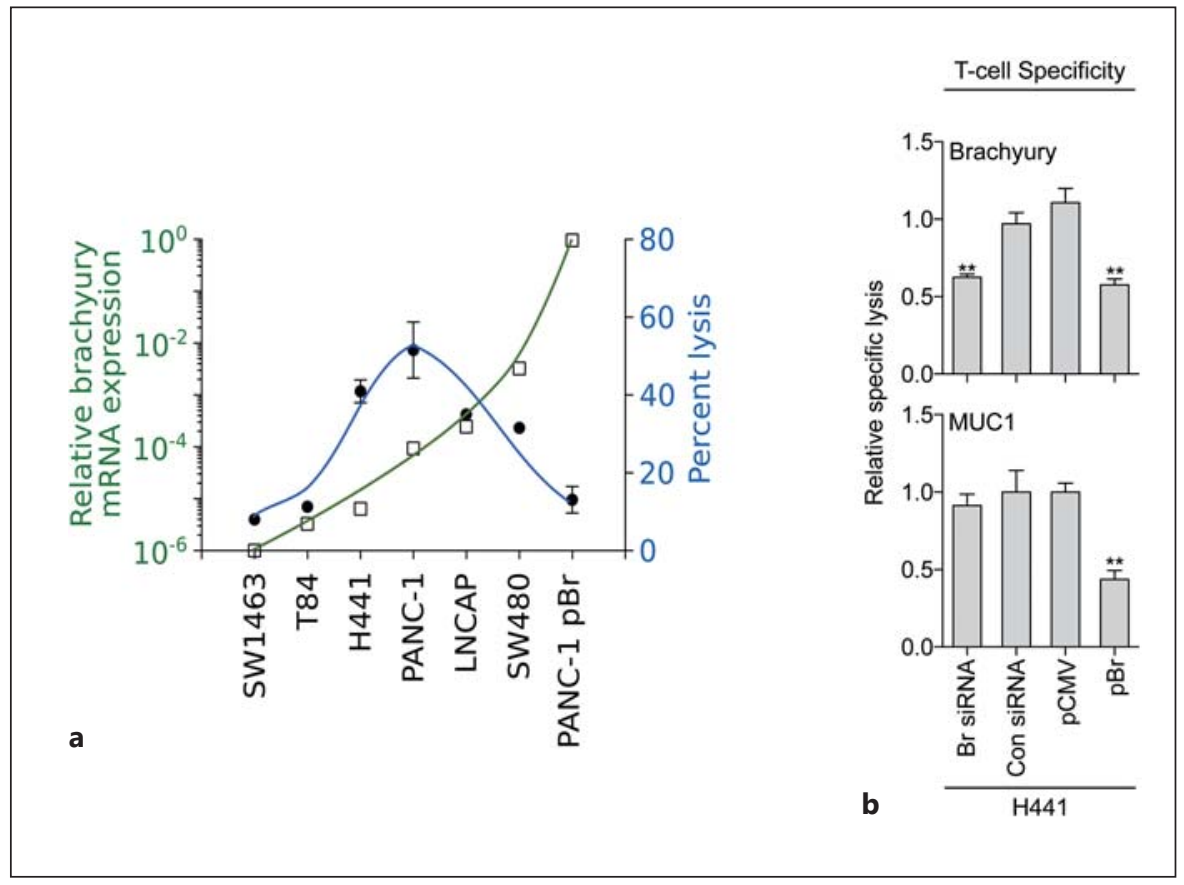

cific T cells (Fig. 1b, upper panel). H441 cells transfected to overexpress or silenced for brachyury expression exhibited a decreased level of lysis compared to the corresponding control cells (pCMV and Con siRNA, respectively), thus recapitulating the bell-shape response curve as shown in Figure 1a. Interestingly, H441 cells are also positive for the tumor antigen MUC1. While the silencing of brachyury did not modify the ability of MUC-1-specific $\mathrm{T}$ cells to lyse the target cells, there was a reduced lysis of brachyury-overexpressing cancer cells. The phenomenon was further studied in detail utilizing multiple isogenic tumor cell pairs with low versus high levels of brachyury, as reported in Hamilton et al. [2014], with brachyury-high cells being less sensitive to the lytic activity of immune effector cells compared to brachyury-intermediate/low cells. The impairment of immune-mediated lysis, however, was also extended to antigen-independent lysis mediated by innate natural killer (NK) or lymphokine-activated killer cells, as well as to lysis mediated by other antigen-specific T cells, even in the presence of normal levels of the corresponding target antigen(s) [Hamilton et al., 2014].

Mechanistic studies demonstrated that the resistance of tumor cells with very high levels of brachyury is due to inefficient caspase-dependent apoptotic death. This apoptotic defect takes place even in the presence of normal levels of fully activated effector caspases, and is man- ifested as the absence of degradation of nuclear lamins due to the loss of the cell cycle-associated kinase CDK1 (Fig. 2). In tumor cells with very high brachyury levels, the levels of CDK1 protein are reduced and, as a consequence, the lamins are not properly phosphorylated, thus precluding the degradation of the nuclear lamina during apoptosis. Importantly, $\mathrm{T}$ cells can lyse tumor cells by caspase-dependent and/or caspase-independent mechanisms, the latter involving perforin/granzymes that can directly cleave nuclear lamins in the presence of apoptotic defects involving caspases. The study of mechanisms of resistance associated with very high levels of brachyury in tumor cells indicated that their lysis could still take place if the effector cells utilize the perforin/granzyme pathway (Fig. 2) [Hamilton et al., 2014].

\section{Rationale for Combinations of Brachyury-Based Cancer Vaccines and Other Antitumor Therapies}

Based on the understanding that tumor cells with very high levels of brachyury may be resistant to immune attack, 2 different strategies are being devised that could potentially be used to improve the outcome of brachyurybased immunotherapies: (1) to repair the apoptotic defect in tumor cells with very high levels of brachyury, and (2) to decrease the expression of brachyury to an optimum 


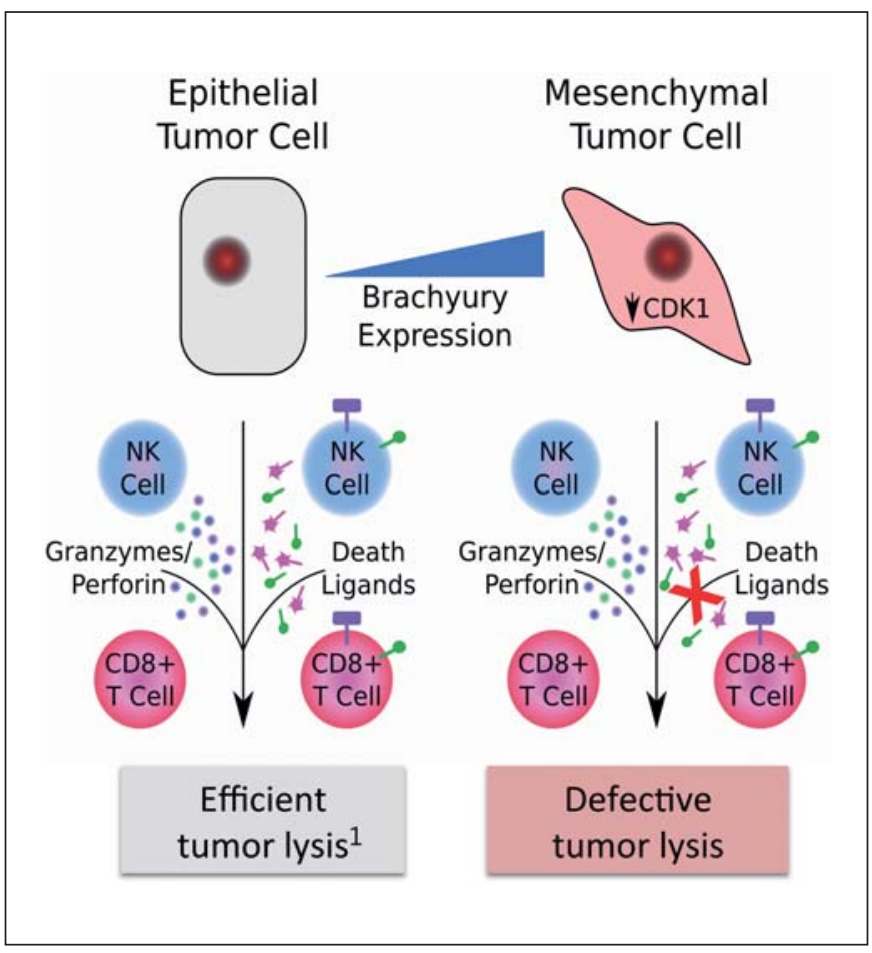

Fig. 2. Proposed model of immune resistance mediated by the expression of very high levels of brachyury in carcinoma cells. Tumor cells with low levels of brachyury can be efficiently lysed by immune effector cells (NK, CD8+ T cells) via the activity of granzymes/perforin (which mediate cell death by caspase-dependent and independent mechanisms) as well as via the activation of cell death receptor/ligand-mediated apoptosis that is exclusively dependent on caspase-mediated mechanisms. ${ }^{1}$ In the particular case of brachyury-specific effector T cells, the absence or very low levels of the antigen brachyury will preclude lysis. In tumor cells with very high levels of brachyury, the death receptor/ligand-mediated apoptotic pathways are not functional due to the loss of CDK1 and subsequent defect on nuclear lamin degradation. While granzyme/ perforin pathways are still able to mediate the lysis of brachyuryhigh tumor cells via activation of caspase-independent pathways, the overall killing of brachyury-high tumor cells is defective.

level where enhanced tumor immune susceptibility could be achieved.

As the decreased apoptosis due to very high levels of brachyury expression is due to decreased CDK1 protein levels, restoration of CDK1 activity in the tumor could potentially allow nuclear apoptosis to take place in response to immune effector cells. One strategy to achieve this objective is via the inhibition of WEE1, a cell cycle kinase that normally inactivates CDK1 by phosphorylating Tyr15 [Ottaviano and Gerace, 1985]. A specific small molecule inhibitor of WEE1, designated AZD1775, is currently in phase I and II clinical trials for the treatment of multiple

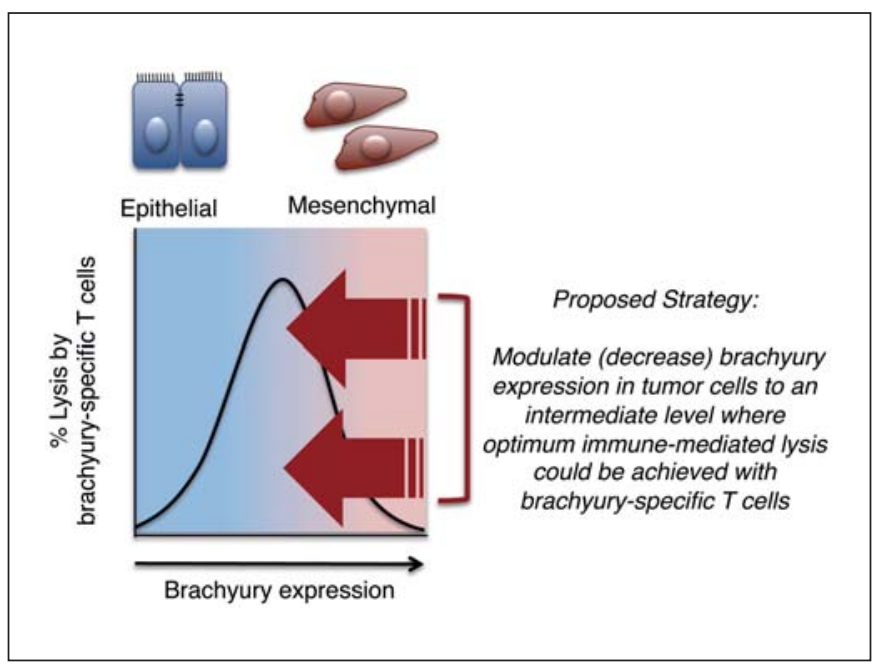

Fig. 3. Proposed model of brachyury reduction as a strategy to improve the lysis of tumor cells by brachyury-based (and other) immune-mediated approaches. tumor types, with demonstrated clinical activity as a single agent [Do et al., 2015]. AZD1775 has been shown to synergize with chemotherapy in preclinical models in vivo with p53-deficient human cancer xenografts [Rajeshkumar et al., 2011]. The inhibitor has also been used in preclinical studies in vitro to improve the lysis of brachyuryhigh tumor cells in response to antigen-specific T cells, NK cells, and the immune mediator TRAIL (tumor necrosis factor-related apoptosis-inducing ligand) via the restoration of caspase-mediated lysis [Hamilton et al., 2014]. Based on these results, AZD1775 could also potentially be used in combination with brachyury-based cancer vaccines to improve the lysis of brachyury-high tumor cells.

The paradoxical observation that tumor lysis may decrease in the presence of very high levels of brachyury led to the model postulated in Figure 3. In accordance with this model, reducing the expression of brachyury in cancer cells to an intermediate level may improve the chances of an antitumor immune response to effectively mediate tumor rejection following vaccination. Additionally, decreasing the expression of brachyury could further improve the outcome of additional therapies as our experiments have demonstrated a central role for the transcription factor brachyury in the phenomenon of tumor resistance to various drugs. Current investigations are focused on elucidating strategies that could reduce the level of brachyury and potentially be used in combination with immunotherapies in future clinical studies. 


\section{Concluding Remarks}

Progress in elucidating the role of tumor EMT in cancer progression and, in particular, in resistance to therapies will help the design of novel therapeutics better suited to addressing the problem of metastatic disease. Immunotherapy has the advantage of eliciting a long-term memory anticancer immune response that could exert tumor control even after the cessation of treatment. Among various immunotherapeutic approaches, cancer vaccines directed against brachyury, an essential driver of EMT, constitute an attractive therapeutic strategy, as cancer vaccines have no associated toxicities and are thus ideal for use in combinatorial approaches [Schlom et al., 2014]. The understanding that tumor plasticity mediated by the process of EMT may enhance resistance to immune attack justifies the future investigation of approaches aimed at reducing EMT in an attempt to improve the outcome of cancer vaccines against drivers of the process.

\section{Acknowledgements}

This research was supported by funds of the NIH Intramural Research Program, National Cancer Institute, Center for Cancer Research.

\section{References}

Akalay, I., B. Janji, M. Hasmim, M.Z. Noman, F. Andre, P. De Cremoux, P. Bertheau, C. Badoual, P. Vielh, A.K. Larsen, M. Sabbah, T.Z. Tan, J.H. Keira, N.T. Hung, J.P. Thiery, F. Mami-Chouaib, S. Chouaib (2013a) Epithelialto-mesenchymal transition and autophagy induction in breast carcinoma promote escape from T-cell-mediated lysis. Cancer Res 73: 2418-2427.

Akalay, I., B. Janji, M. Hasmim, M.Z. Noman, J.P Thiery, F. Mami-Chouaib, S. Chouaib (2013b) EMT impairs breast carcinoma cell susceptibility to CTL-mediated lysis through autophagy induction. Autophagy 9: 1104-1106.

Barresi, V., A. Ieni, G. Branca, G. Tuccari (2014) Brachyury: a diagnostic marker for the differential diagnosis of chordoma and hemangioblastoma versus neoplastic histological mimickers. Dis Markers 2014: 514753.

Barresi, V., E. Vitarelli, G. Branca, M. Antonelli, F. Giangaspero, G. Barresi (2012) Expression of brachyury in hemangioblastoma: potential use in differential diagnosis. Am J Surg Pathol 36:1052-1057.

Bilusic, M., C.R. Heery, P.M. Arlen, M. Rauckhorst, D. Apelian, K.Y. Tsang, J.A. Tucker, C. Jochems, J. Schlom, J.L. Gulley, R.A. Madan (2014) Phase I trial of a recombinant yeastCEA vaccine (GI-6207) in adults with metastatic CEA-expressing carcinoma. Cancer Immunol Immunother 63: 225-234.

Borghaei, H., L. Paz-Ares, L. Horn, D.R. Spigel, M. Steins, N.E. Ready, L.Q. Chow, E.E. Vokes, E. Felip, E. Holgado, F. Barlesi, M. Kohlhaufl, O. Arrieta, M.A. Burgio, J. Fayette, H. Lena, E. Poddubskaya, D.E. Gerber, S.N. Gettinger, C.M. Rudin, N. Rizvi, L. Crino, G.R. Blumenschein, Jr., S.J. Antonia, C. Dorange, C.T. Harbison, F. Graf Finckenstein, J.R. Brahmer (2015) Nivolumab versus Docetaxel in advanced nonsquamous non-small-cell lung cancer. N Engl J Med 373: 1627-1639.
Byers, L.A., L. Diao, J. Wang, P. Saintigny, L. Girard, M. Peyton, et al. (2013) An epithelialmesenchymal transition gene signature predicts resistance to EGFR and PI3K inhibitors and identifies Axl as a therapeutic target for overcoming EGFR inhibitor resistance. Clin Cancer Res 19: 279-290.

Chaft, J.E., A. Litvak, M.E. Arcila, P. Patel, S.P. D’Angelo, L.M. Krug, V. Rusch, A. Mattson, C. Coeshott, B. Park, D.M. Apelian, M.G. Kris, C.G. Azzoli (2014) Phase II study of the GI-4000 KRAS vaccine after curative therapy in patients with stage I-III lung adenocarcinoma harboring a KRAS G12C, G12D, or G12V mutation. Clin Lung Cancer 15: 405410.

Creighton, C.J., X. Li, M. Landis, J.M. Dixon, V.M. Neumeister, A. Sjolund, D.L. Rimm, H. Wong, A. Rodriguez, J.I. Herschkowitz, C. Fan, X. Zhang, X. He, A. Pavlick, M.C. Gutierrez, L. Renshaw, A.A. Larionov, D. Faratian, S.G. Hilsenbeck, C.M. Perou, M.T. Lewis, J.M. Rosen, J.C. Chang (2009) Residual breast cancers after conventional therapy display mesenchymal as well as tumor-initiating features. Proc Natl Acad Sci USA 106: 13820-13825.

Do, K., D. Wilsker, J. Ji, J. Zlott, T. Freshwater, R.J. Kinders, J. Collins, A.P. Chen, J.H. Doroshow, S. Kummar (2015) Phase I study of single-agent AZD1775 (MK-1775), a Wee1 kinase inhibitor, in patients with refractory solid tumors. J Clin Oncol 33: 3409-3415.

$\mathrm{Du}, \mathrm{R}$., S. Wu, X. Lv, H. Fang, S. Wu, J. Kang (2014) Overexpression of brachyury contributes to tumor metastasis by inducing epithelial-mesenchymal transition in hepatocellular carcinoma. J Exp Clin Cancer Res 33: 105.

Edwards, Y.H., W. Putt, K.M. Lekoape, D. Stott, M. Fox, D.A. Hopkinson, J. Sowden (1996) The human homolog $T$ of the mouse $T$ (Brachyury) gene; gene structure, cDNA sequence, and assignment to chromosome 6q27. Genome Res 6: 226-233.
Fernando, R.I., M.D. Castillo, M. Litzinger, D.H. Hamilton, C. Palena (2011) IL-8 signaling plays a critical role in the epithelial-mesenchymal transition of human carcinoma cells. Cancer Res 71: 5296-5306.

Fernando, R.I., M. Litzinger, P. Trono, D.H. Hamilton, J. Schlom, C. Palena (2010) The Tbox transcription factor Brachyury promotes epithelial-mesenchymal transition in human tumor cells. J Clin Invest 120: 533-544.

Gabitzsch, E.S., K.Y. Tsang, C. Palena, J.M. David, M. Fantini, A. Kwilas, A.E. Rice, Y. Latchman, J.W. Hodge, J.L. Gulley, R.A. Madan, C.R. Heery, J.P. Balint Jr., F.R. Jones, J. Schlom (2015) The generation and analyses of a novel combination of recombinant adenovirus vaccines targeting three tumor antigens as an immunotherapeutic. Oncotarget 6: 3134431359.

Gabitzsch, E.S., Y. Xu, J.P. Balint, Jr., Z.C. Hartman, H.K. Lyerly, F.R. Jones (2010) Anti-tumor immunotherapy despite immunity to adenovirus using a novel adenoviral vector Ad5 [E1-, E2b-]-CEA. Cancer Immunol Immunother 59: 1131-1135.

Gabitzsch, E.S., Y. Xu, L.H. Yoshida, J. Balint, R.B. Gayle, A. Amalfitano, F.R. Jones (2009) A preliminary and comparative evaluation of a novel Ad5 [E1-, E2b-] recombinant-based vaccine used to induce cell mediated immune responses. Immunol Lett 122: 44-51.

Garon, E.B., N.A. Rizvi, R. Hui, N. Leighl, A.S. Balmanoukian, J.P. Eder, A. Patnaik, C. Aggarwal, M. Gubens, L. Horn, E. Carcereny, M.J. Ahn, E. Felip, J.S. Lee, M. D. Hellmann, O. Hamid, J.W. Goldman, J.C. Soria, M. Dolled-Filhart, R.Z. Rutledge, J. Zhang, J.K. Lunceford, R. Rangwala, G.M. Lubiniecki, C. Roach, K. Emancipator, L. Gandhi (2015) Pembrolizumab for the treatment of nonsmall-cell lung cancer. N Engl J Med 372: 2018-2028. 
Hamilton, D.H., R.I. Fernando, J. Schlom, C. Palena (2015) Aberrant expression of the embryonic transcription factor brachyury in human tumors detected with a novel rabbit monoclonal antibody. Oncotarget 6: 48534862.

Hamilton, D.H., B. Huang, R.I. Fernando, K.Y. Tsang, C. Palena (2014) WEE1 inhibition alleviates resistance to immune attack of tumor cells undergoing epithelial-mesenchymal transition. Cancer Res 74: 2510-2519.

Hamilton, D.H., M. T. Litzinger, A. Jales, B. Huang, R.I. Fernando, J.W. Hodge, A. Ardiani, D. Apelian, J. Schlom, C. Palena (2013) Immunological targeting of tumor cells undergoing an epithelial-mesenchymal transition via a recombinant brachyury-yeast vaccine. Oncotarget 4: 1777-1790.

Hanahan, D., R.A. Weinberg (2011) Hallmarks of cancer: the next generation. Cell 144: 646674.

Haro, A., T. Yano, M. Kohno, T. Yoshida, T. Koga, T. Okamoto, M. Takenoyama, Y. Maehara (2013) Expression of Brachyury gene is a significant prognostic factor for primary lung carcinoma. Ann Surg Oncol 20(suppl 3): S509-S516.

Heery, C.R., B.H. Singh, M. Rauckhorst, J.L. Marte, R.N. Donahue, I. Grenga, T.C. Rodell, W. Dahut, P.M. Arlen, R.A. Madan, J. Schlom, J.L. Gulley (2015) Phase I trial of a yeast-based therapeutic cancer vaccine (GI6301) targeting the transcription factor brachyury. Cancer Immunol Res 3: 12481256.

Herrmann, B.G., S. Labeit, A. Poustka, T.R. King, H. Lehrach (1990) Cloning of the T gene required in mesoderm formation in the mouse. Nature 343: 617-622.

Huang, B., J.R. Cohen, R.I. Fernando, D.H. Hamilton, M. T. Litzinger, J.W. Hodge, C. Palena (2013) The embryonic transcription factor Brachyury blocks cell cycle progression and mediates tumor resistance to conventional antitumor therapies. Cell Death Dis 4: e682.

Imajyo, I., T. Sugiura, Y. Kobayashi, M. Shimoda, K. Ishii, N. Akimoto, N. Yoshihama, I. Kobayashi, Y. Mori (2012) T-box transcription factor Brachyury expression is correlated with epithelial-mesenchymal transition and lymph node metastasis in oral squamous cell carcinoma. Int J Oncol 41: 1985-1995.

Kalluri, R., R.A. Weinberg (2009) The basics of epithelial-mesenchymal transition. J Clin Invest 119: 1420-1428.

Kilic, N., S. Feldhaus, E. Kilic, P. Tennstedt, D. Wicklein, R. Wasielewski, C. Viebahn, H. Kreipe, U. Schumacher (2011) Brachyury expression predicts poor prognosis at early stages of colorectal cancer. Eur J Cancer 47: 10801085.

Kim, H.P., S.W. Han, S.H. Song, E.G. Jeong, M.Y. Lee, D. Hwang, S.A. Im, Y.J. Bang, T.Y. Kim (2014) Testican-1-mediated epithelial-mesenchymal transition signaling confers acquired resistance to lapatinib in HER2-positive gastric cancer. Oncogene 33: 3334-3341.
Kispert, A., B.G. Herrmann (1993) The Brachyury gene encodes a novel DNA binding protein. EMBO J 12: 3211-3220.

Kispert, A., B. Koschorz, B.G. Herrmann (1995) The $\mathrm{T}$ protein encoded by Brachyury is a tissue-specific transcription factor. EMBO J 14: 4763-4772.

Kudo-Saito, C., H. Shirako, T. Takeuchi, Y. Kawakami (2009) Cancer metastasis is accelerated through immunosuppression during Snail-induced EMT of cancer cells. Cancer Cell 15: 195-206

Kurrey, N.K., S.P. Jalgaonkar, A.V. Joglekar, A.D. Ghanate, P.D. Chaskar, R.Y. Doiphode, S.A. Bapat (2009) Snail and slug mediate radioresistance and chemoresistance by antagonizing p53-mediated apoptosis and acquiring a stem-like phenotype in ovarian cancer cells. Stem Cells 27: 2059-2068.

Larocca, C., J.R. Cohen, R.I. Fernando, B. Huang, D.H. Hamilton, C. Palena (2013) An autocrine loop between TGF- $\beta 1$ and the transcription factor brachyury controls the transition of human carcinoma cells into a mesenchymal phenotype. Mol Cancer Ther 12: 18051815.

Madan, R.A., M. Bilusic, C. Heery, J. Schlom, J.L. Gulley (2012) Clinical evaluation of TRICOM vector therapeutic cancer vaccines. Semin Oncol 39: 296-304.

Madan, R. A., M. Mohebtash, P.M. Arlen, M. Vergati, M. Rauckhorst, S.M. Steinberg, K. Y. Tsang, D.J. Poole, H.L. Parnes, J.J. Wright, W.L. Dahut, J. Schlom, J.L. Gulley (2012) Ipilimumab and a poxviral vaccine targeting prostate-specific antigen in metastatic castration-resistant prostate cancer: a phase 1 doseescalation trial. Lancet Oncol 13: 501-508.

Mayr, A., K. Danner (1978) Vaccination against pox diseases under immunosuppressive conditions. Dev Biol Stand 41: 225-234.

Miettinen, M., Z. Wang, J. Lasota, C. Heery, J. Schlom, C. Palena (2015) Nuclear brachyury expression is consistent in chordoma, common in germ cell tumors and small cell carcinomas, and rare in other carcinomas and sarcomas: an immunohistochemical study of 5,229 cases. Am J Surg Pathol 39: 1305-1312.

Ottaviano, Y., L. Gerace (1985) Phosphorylation of the nuclear lamins during interphase and mitosis. J Biol Chem 260: 624-632.

Palena, C., R. I. Fernando, D. H. Hamilton (2014) An immunotherapeutic intervention against tumor progression: targeting a driver of the epithelial-to-mesenchymal transition. Oncoimmunology 3: e27220.

Palena, C., D.E. Polev, K.Y. Tsang, R.I. Fernando, M. Litzinger, L.L. Krukovskaya, A.V. Baranova, A.P. Kozlov, J. Schlom (2007) The human T-box mesodermal transcription factor Brachyury is a candidate target for T-cell-mediated cancer immunotherapy. Clin Cancer Res 13: 2471-2478.
Palena, C., M. Roselli, M.T. Litzinger, P. Ferroni, L. Costarelli, A. Spila, F. Cavaliere, B. Huang, R.I. Fernando, D.H. Hamilton, C. Jochems, K.Y. Tsang, Q. Cheng, H. Kim Lyerly, J. Schlom, F. Guadagni (2014) Overexpression of the EMT driver brachyury in breast carcinomas: association with poor prognosis. J Natl Cancer Inst 106: dju054.

Palena, C., J. Schlom (2010) Vaccines against human carcinomas: strategies to improve antitumor immune responses. J Biomed Biotechnol 2010: 380697.

Pinto, F., N.C. Campanella, L.F. Abrahao-Machado, C. Scapulatempo-Neto, A.T. de Oliveira, M.J. Brito, R.P. Andrade, D.P. Guimaraes, R.M. Reis (2015) The embryonic Brachyury transcription factor is a novel biomarker of GIST aggressiveness and poor survival. Gastric Cancer 19: 651-659.

Pinto, F., N. Pertega-Gomes, M.S. Pereira, J.R. Vizcaino, P. Monteiro, R.M. Henrique, F. Baltazar, R.P. Andrade, R.M. Reis (2014) Tbox transcription factor Brachyury is associated with prostate cancer progression and aggressiveness. Clin Cancer Res 20: 4949-4961.

Powles, T., J.P. Eder, G.D. Fine, F.S. Braiteh, Y. Loriot, C. Cruz, J. Bellmunt, H.A. Burris, D.P Petrylak, S.L. Teng, X. Shen, Z. Boyd, P.S. Hegde, D.S. Chen, N.J. Vogelzang (2014) MPDL3280A (anti-PD-L1) treatment leads to clinical activity in metastatic bladder cancer. Nature 515: 558-562.

Rajeshkumar, N.V., E. De Oliveira, N. Ottenhof, J. Watters, D. Brooks, T. Demuth, S.D. Shumway, S. Mizuarai, H. Hirai, A. Maitra, M. Hidalgo (2011) MK-1775, a potent Wee1 inhibitor, synergizes with gemcitabine to achieve tumor regressions, selectively in p53-deficient pancreatic cancer xenografts. Clin Cancer Res 17: 2799-2806.

Ribas, A. (2015) Adaptive immune resistance: how cancer protects from immune attack. Cancer Discov 5: 915-919.

Roselli, M., R.I. Fernando, F. Guadagni, A. Spila, J. Alessandroni, R. Palmirotta, L. Costarelli, M. Litzinger, D. Hamilton, B. Huang, J. Tucker, K.Y. Tsang, J. Schlom, C. Palena (2012) Brachyury, a driver of the epithelial-mesenchymal transition, is overexpressed in human lung tumors: an opportunity for novel interventions against lung cancer. Clin Cancer Res 18: 3868-3879.

Schlom, J., J.W. Hodge, C. Palena, K.Y. Tsang, C. Jochems, J.W. Greiner, B. Farsaci, R.A. Madan, C.R. Heery, J.L. Gulley (2014) Therapeutic cancer vaccines. Adv Cancer Res 121: 67-124.

Shao, C., J. Zhang, J. Fu, F. Ling (2015) The potential role of Brachyury in inducing epithelial-to-mesenchymal transition (EMT) and HIF-1 $\alpha$ expression in breast cancer cells. Biochem Biophys Res Commun 467: 1083-1089.

Shin, D. S., A. Ribas (2015) The evolution of checkpoint blockade as a cancer therapy: what's here, what's next? Curr Opin Immunol 33C: 23-35. 
Tan, T.Z., Q.H. Miow, Y. Miki, T. Noda, S. Mori, R.Y. Huang, J.P. Thiery (2014) Epithelialmesenchymal transition spectrum quantification and its efficacy in deciphering survival and drug responses of cancer patients. EMBO Mol Med 6: 1279-1293.

Tang, X., L. Jing, J. Chen (2012) Changes in the molecular phenotype of nucleus pulposus cells with intervertebral disc aging. PLoS One 7: e52020.

Thiery, J.P. (2002) Epithelial-mesenchymal transitions in tumour progression. Nat Rev Cancer 2: 442-454.

Thiery, J.P. (2003) Epithelial-mesenchymal transitions in development and pathologies. Curr Opin Cell Biol 15: 740-746.

Thomson, S., E. Buck, F. Petti, G. Griffin, E. Brown, N. Ramnarine, K.K. Iwata, N. Gibson, J.D. Haley (2005) Epithelial to mesenchymal transition is a determinant of sensitivity of non-small-cell lung carcinoma cell lines and xenografts to epidermal growth factor receptor inhibition. Cancer Res 65:9455-9462.

Thomson, S., F. Petti, I. Sujka-Kwok, D. Epstein, J.D. Haley (2008) Kinase switching in mesenchymal-like non-small cell lung cancer lines contributes to EGFR inhibitor resistance through pathway redundancy. Clin Exp Metastasis 25:843-854.
Tirabosco, R., D.C. Mangham, A.E. Rosenberg, S. Vujovic, K. Bousdras, S. Pizzolitto, G. De Maglio, M.A. den Bakker, L. Di Francesco, R.K. Kalil, N.A. Athanasou, P. O'Donnell, E.F. McCarthy, A.M. Flanagan (2008) Brachyury expression in extra-axial skeletal and soft tissue chordomas: a marker that distinguishes chordoma from mixed tumor/myoepithelio$\mathrm{ma} /$ parachordoma in soft tissue. Am J Surg Pathol 32: 572-580.

Topalian, S.L., M. Sznol, D.F. McDermott, H.M. Kluger, R.D. Carvajal, W.H. Sharfman, J.R. Brahmer, D.P. Lawrence, M.B. Atkins, J.D. Powderly, P.D. Leming, E.J. Lipson, I. Puzanov, D.C. Smith, J.M. Taube, J.M. Wigginton, G.D. Kollia, A. Gupta, D. M. Pardoll, J.A. Sosman, F.S. Hodi (2014) Survival, durable tumor remission, and long-term safety in patients with advanced melanoma receiving nivolumab. J Clin Oncol 32: 1020-1030.

Tucker, J.A., C. Jochems, B. Boyerinas, J. Fallon, J.W. Greiner, C. Palena, T.C. Rodell, J. Schlom, K.Y. Tsang (2014) Identification and characterization of a cytotoxic T-lymphocyte agonist epitope of brachyury, a transcription factor involved in epithelial to mesenchymal transition and metastasis. Cancer Immunol Immunother 63: 1307-1317.

Vega, S., A.V. Morales, O.H. Ocana, F. Valdes, I. Fabregat, M.A. Nieto (2004) Snail blocks the cell cycle and confers resistance to cell death. Genes Dev 18: 1131-1143.

Vujovic, S., S. Henderson, N. Presneau, E. Odell, T.S. Jacques, R. Tirabosco, C. Boshoff, A.M. Flanagan (2006) Brachyury, a crucial regulator of notochordal development, is a novel biomarker for chordomas. J Pathol 209: 157165.
Whiteside, T.L. (2009) Tricks tumors use to escape from immune control. Oral Oncol 45: e119-123.

Wilkinson, D.G., S. Bhatt, B.G. Herrmann (1990) Expression pattern of the mouse $\mathrm{T}$ gene and its role in mesoderm formation. Nature 343 : 657-659.

Xu, K., B. Liu, Y. Liu (2015) Impact of Brachyury on epithelial-mesenchymal transitions and chemosensitivity in non-small cell lung cancer. Mol Med Rep 12: 995-1001.

Yang, X.R., D. Ng, D.A. Alcorta, N.J. Liebsch, E. Sheridan, S. Li, A.M. Goldstein, D.M. Parry, M.J. Kelley (2009) T (brachyury) gene duplication confers major susceptibility to familial chordoma. Nat Genet 41: 1176-1178.

Yu, M., A. Bardia, B.S. Wittner, S.L. Stott, M.E. Smas, D.T. Ting, S.J. Isakoff, J.C. Ciciliano, M.N. Wells, A.M. Shah, K.F. Concannon, M.C. Donaldson, L.V. Sequist, E. Brachtel, D. Sgroi, J. Baselga, S. Ramaswamy, M. Toner, D.A. Haber, S. Maheswaran (2013) Circulating breast tumor cells exhibit dynamic changes in epithelial and mesenchymal composition. Science 339: 580-584.

Zitvogel, L., A. Tesniere, G. Kroemer (2006) Cancer despite immunosurveillance: immunoselection and immunosubversion. Nat Rev Immunol 6: 715-727. 